\title{
Nanosized Optoelectronic Devices Based on Photoactivated Proteins
}

\author{
Alice Dimonte, ${ }^{*}$ Stefano Frache, ${ }^{\S}$, Victor Erokhin, ${ }^{\|}$Gianluca Piccinini, ${ }^{\S}$ Danilo Demarchi, ${ }^{\dagger}$, \\ Francesco Milano, ${ }^{\perp}$ Giovanni De Micheli, ${ }^{\ddagger}$ and Sandro Carrara ${ }^{\ddagger}$ \\ ${ }^{\dagger}$ Fondazione Istituto Italiano di Tecnologia, IIT@Polito Center, Torino, Italy \\ ${ }^{\ddagger}$ Ecole Polytechnique Federale de Lausanne (EPFL), Lausanne, VD, Switzerland \\ ${ }^{\S}$ Department of Electronics and Telecomunications, Politecnico di Torino, Torino, Italy \\ "CNR-IMEMf Physics Department, University of Parma, Parma, Italy \\ ${ }^{\perp}$ CNR-IPCF Bari Division, c/o Chemistry Department, University of Bari, Bari, Italy
}

ABSTRACT: Molecular nanoelectronics is attracting much attention, because of the possibility to add functionalities to silicon-based electronics by means of intrinsically nanoscale biological or organic materials. The contact point between active molecules and electrodes must present, besides nanoscale size, a very low resistance. To realize Metal-Molecule-Metal junctions it is, thus, mandatory to be able to control the formation of useful nanometric contacts. The distance between the electrodes has to be of the same size of the molecule being put in between. Nanogaps technology is a perfect fit to fulfill this requirement. In this work, nanogaps between gold electrodes have been used to develop optoelectronic devices based on photoactive proteins. Reaction Centers (RC) and Bacteriorhodopsin (BR) have been inserted in nanogaps by drop casting. Electrical characterizations of the obtained structures were performed. It has been demonstrated that these nanodevices working principle is based on charge separation and photovoltage response. The former is induced by the application of a proper voltage on the RC, while the latter comes from the activation of BR by light of appropriate wavelengths.

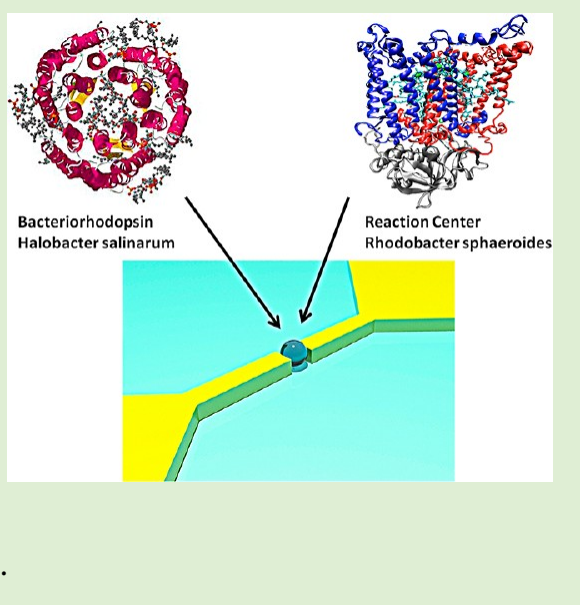

\section{INTRODUCTION}

Molecular electronics is appealing to researchers because of its possible exploitation as a viable alternative to silicon-based electronic devices. The main underlying idea is to use single molecules as active elements in nanodevices. ${ }^{1}$ As a consequence, the proper realization of a molecule-electrode contact is a crucial issue. ${ }^{2,3}$ Among the structures that enable us to perform electrical characterization at molecular level there are last-generation probe microscopes, such as AFM conducting tip, ${ }^{4}$ nanopores structures, ${ }^{5}$ and nanogaps structures. ${ }^{6,7}$ The nanogaps, in particular, seem promising because they do not require feedback to maintain the mutual arrangement (comparing with conducting tip AFM) and are less stochastic with respect to nanopores structures. Moreover, these fractures in metal wire can have dimensions comparable to the size of the molecule that has to be inserted in between them. Several techniques can be applied to nanogap fabrication: mechanically broken $^{8}$ or positioned junctions, ${ }^{9-11}$ nanoscale lithography by Synchrotron radiation sources, ${ }^{12}$ electrochemical deposition and etching, ${ }^{13-15}$ and electromigration. ${ }^{16}$ None of these techniques is presently able to give precise control as to the final gap size.

This work focuses on the electromigration ${ }^{17}$ approach that has demonstrated several useful characteristics. It is costeffective because of the relatively low complexity of the required equipment. It can be embedded into a lab-on-chip system, thus exploiting the possibility to tailor the gap formation process by means of a digital loop control system. To this end, it just requires a conventional microchip fabrication process. Nanogaps below $10 \mathrm{~nm}$ are commonly fabricated with this approach. Electromigration is a phenomenon involving net mass transport of a conducting material: due to an applied electric field, electrons transfer momentum to the ions that constitute the crystalline lattice of the material. Quantitatively, the process can be described by a phenomenological law, known as Black's law, ${ }^{18}$ that relates electromigration to the current density in the wire and to the absolute temperature at which the phenomenon takes place. To realize the gap size of just a few nanometers, an accurate control of both probe shape and applied voltage is mandatory. ${ }^{19}$

$\mathrm{Xu}$ et al. showed a new way to exploit nanogap probes ${ }^{20}$ to gain energy from photosynthetic proteins from bacteria. These bacteria are able to convert light energy into biochemical energy that in nature allows the survival of the microorganism they belong to. Here their ability is exploited to realize metalmolecule-metal junctions, which can be thought of as optoelectronic transistors. ${ }^{21,22}$

Photosynthetic reaction centers (RCs) from the purple bacteria Rhodobacter sphaeroides are integral transmembrane proteins, ${ }^{23}$ which are able to convert the solar energy in

Received: May 7, 2012

Revised: October 3, 2012

Published: October 9, 2012 
chemical energy in the form of a charge-separated state. In vivo, a complex photocycle, ${ }^{24}$ also involving other proteins, leads to a proton translocation across the plasmatic membrane, which fuels the bacterial metabolism. Conversely, in isolated RC, the photoinduced charge-separated state decays in the dark through a charge recombination reaction. ${ }^{25}$

Bacteriorhodopsins are chromoproteins localized in the purple membrane of the bacterium Halobacter salinarum ${ }^{26}$ where they are organized as an array of trimers semicrystallines, each consisting in $3 \mathrm{BR}$ proteins connected by a 3D symmetry axis standing as a rigid element of a highly ordered lattice. ${ }^{27}$ Moreover, the purple membrane belongs to the group of plasmatic membranes that can transfer energy. As in the case of RC from Rhodobacter sphaeroides, the luminous energy is employed for the life of the bacterium. ${ }^{28}$ The incoming radiation has to be in the green visible light range, that is, $\sim 570$ $\mathrm{nm}$ wavelength, to obtain a reaction in the BR. Photoelectrical phenomenon in BR is highly different from the one caused by irradiation in RC. The molecules of BR work as light-driven proton pumps: during the photocycle, a transmembrane proton gradient is generated. However, the light-induced cycle in RC consists of the electron transfer across its membrane because of the presence of four types of cofactors (BacterioPheophytins, BacterioChlorophylls, Quinones, and a nonheme $\mathrm{Fe}^{2+}$ ), as described in refs 29 and 30.

Studies of photosensitive proteins, such as BR and RC, have a rather long history dating back to the 1970s to 1980s. Even if the investigations at single-molecule level were not possible at that time, particular interest was raised by the study of Langmuir-Blodgett films of these molecules in sandwich structures, where the thickness of the active layer was down to one monolayer. In particular, the technique was used to study $\mathrm{RC}^{31-42}$ and $\mathrm{BR}^{43-47}$

In recent years, because of technology enhancement, it was possible to develop more complex devices and methods for characterizing these proteins. Photoelectrochemical systems have been designed to realize sensors, as the one produced in ref 48, where RC proteins have been entrapped between metal electrodes or immobilized onto gold chips. ${ }^{49,50}$ Devices made by self-assembled monolayers (SAMs) have been developed by refs 51-53. In ref 54, a conductive atomic force microscopy has been used to characterize RC proteins on SAM-modified gold layer. BR proteins were sandwiched into ITO-PET ${ }^{55}$ or integrated in more complex circuits like $\mathrm{FET}^{56}$ and MOSFET. ${ }^{57} \mathrm{BR}$, as a medium for biomolecular optoelectronics, has been interfaced, especially as a thin-film, solid-state currentcarrying electronic element of metal-molecule-metal system. ${ }^{58}$

An interesting method to activate bacteriorhodopsin-based electrodes has been developed by ref 59 with an enhanced electrical response from the protein thanks to the photonic emission of $\mathrm{CdSe} / \mathrm{ZnS}$ quantum dots, which is absorbed by the bacteriorhodopsin retinal and initiates the proton pumping sequence, resulting in an electrical output from a bacteriorhodopsin-based electrode.

The performances of precursory metal-molecule-metal nanojunctions are here shown through the obtained results that could find application in the aforementioned fields, like sensing, imaging, and solar energy conversion.

\section{MATERIALS AND METHODS}

2.1. Chemicals. RCs were isolated from Rhodobacter sphaeroides strain R26 (obtained from the German Collection of Microorganisms and Cell Cultures) following the procedure illustrated by ref 60 . Protein purity was checked using the ratio of the absorbance at 280 and $802 \mathrm{~nm}$, which was kept below 1.3, whereas the integrity was checked by the ratio of the absorbance at 760 and $865 \mathrm{~nm}$, which was kept close to unity, as shown in Figure 1. The average quinone content

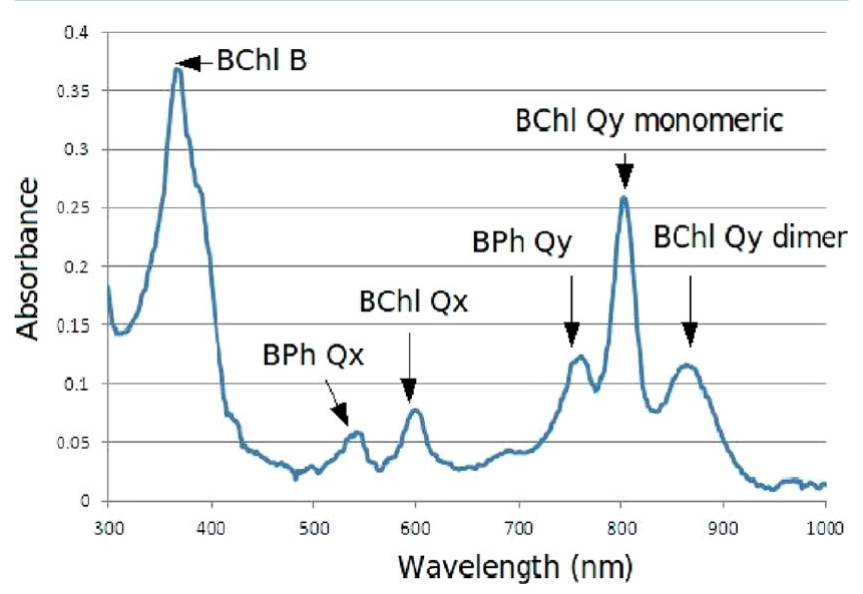

Figure 1. Steady absorption spectra obtained with a Jenway 6415 Spectrometer of $92 \mu \mathrm{M}$ RC solved 1:100 in the $\mathrm{pH} 8.0$ Tris-EDTALDAO buffer.

was 1.8 per RC, and the final concentration of the stocks was $92 \mu \mathrm{M}$. Reagents for solution preparation (Tris, EDTA, LDAO) and Bacteriorhodopsin proteins in the form of purple membrane (B0184) were bought by Sigma Aldrich in Switzerland. Two types of solutions have been prepared: RC proteins were suspended at 0.92 $\mu \mathrm{M}$ final concentration in Tris- $\mathrm{HCl} 15 \mathrm{mM}$, EDTA $1 \mathrm{mM}$, and LDAO $0.1 \%$ at $\mathrm{pH}$ 8.0. Bacteriorhodopsin proteins were solved in deionized water (at pH 5.5 with resistivity of $18 \mathrm{M} \Omega \mathrm{cm}$ ) and inserted in an ultrasound bath for $2 \mathrm{~min}$ for crumbling the purple membranes before deposition; in particular, three types of $\mathrm{BR}$ solutions have been prepared solving $1 \mu \mathrm{g}$ of $\mathrm{BR}$, respectively, in 1,10 , and $100 \mu \mathrm{L}$ of DI water.

2.2. Nanogaps Fabrication. All nanogaps used belong to a family of lab-on-chips made in the CMI@EPFL Lausanne, as the one shown in Figure 2. They have been realized through electromigration, exploiting an "ad hoc" closed loop digital control system implemented in a custom embedded design. A microcontroller monitors the resistance of the gold wire while applying a step-up voltage. As soon as the system detects a steep increase in the resistance, not related to the increment of temperature, it reduces the voltage across the wire of a percentage established by user interface. Then, it iterates the process until the resistance reaches a threshold of $\sim 12.9 \mathrm{k} \Omega$ (i.e., the inverse of the quantized conductance of a gold atom ${ }^{61,62}$ ), at which point it is safe to assume that proper nanogap will be formed because of the dynamics of the control system. Figure 3 shows the process of a nanogap fabrication: different levels of detail are shown about the wire's rising resistance and the activations of the control system.

Gaps sizes were investigated by means of a scanning electron microscope NVision 40 from Zeiss. Images were acquired at $3 \mathrm{kV}$ : such a low voltage is needed to avoid damages to the thin gold layer where the nanogaps are realized, but it still grants adequate image resolution, as visible in Figure 4. After SEM measurements session, the best nanogaps were chosen for the metal-molecule-metal nanojunctions preparation.

2.3. AFM Measurements. Atomic force microscopy XE-70 of Park System was also employed to characterize the metal-moleculemetal junctions. AFM images were acquired working in contact mode. The contact force was kept below $3 \mathrm{nN}$ during the observation to prevent damaging of probes or molecules.

2.4. Photoinduced Electrical Measurements. The photovoltage acquisitions have been performed by means of two different probestations. For RCs, a Karl Suss PM8Manual probe-station with an 

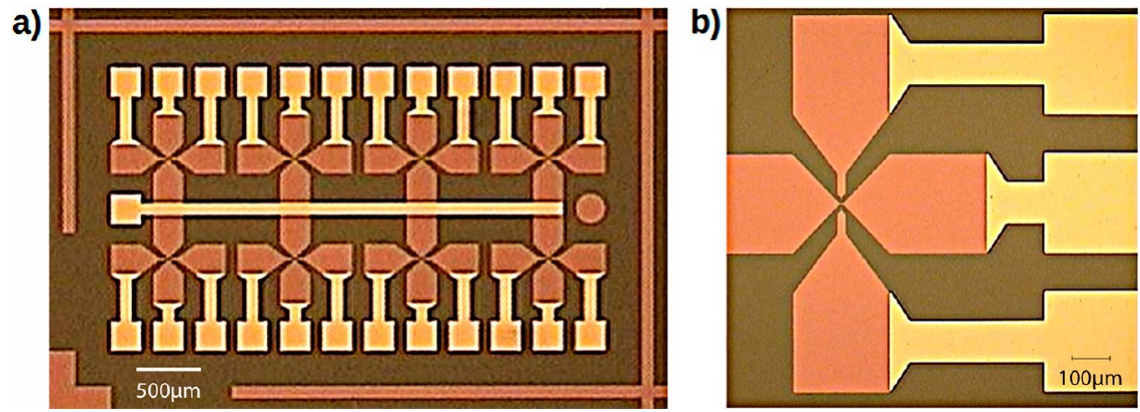

Figure 2. (a) Optical microscope photography of the final chip with the eight probes for the nanogap realization in red (i.e., gold), while the pads for the contact are in yellow (i.e., aluminum). (b) Zoom of one of the probes showing the wire in the horizontal part with two perpendicular elements representing the gates.

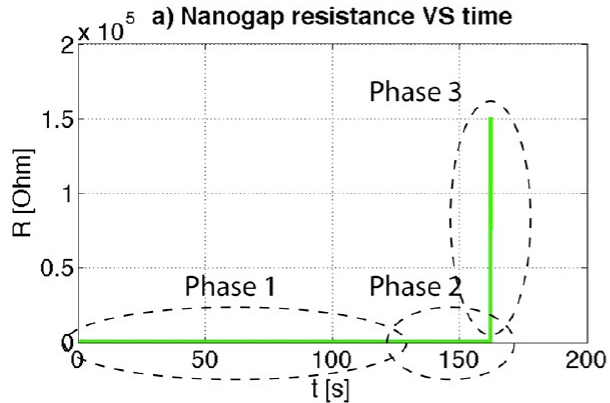

c) High level of detail of nanogap resistance VS time

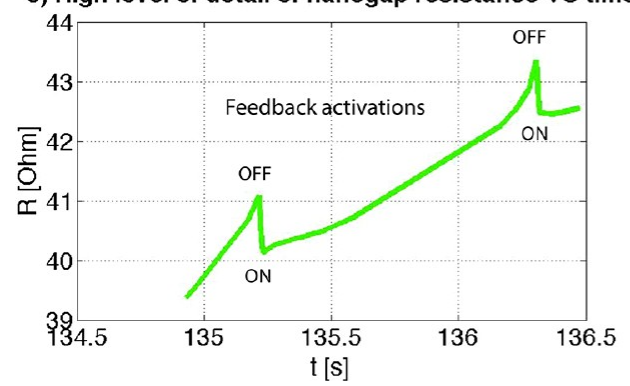

b) Detail of nanogap resistance VS time

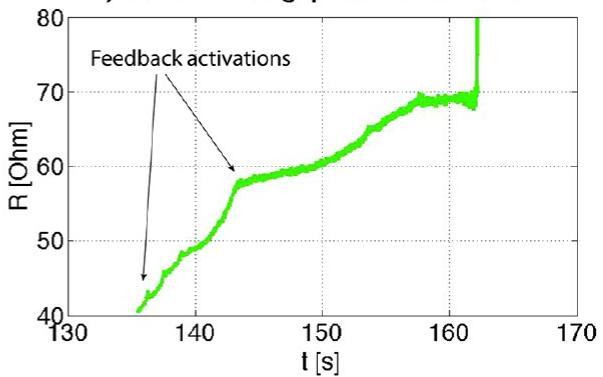

d) Current through the nanogap vS time

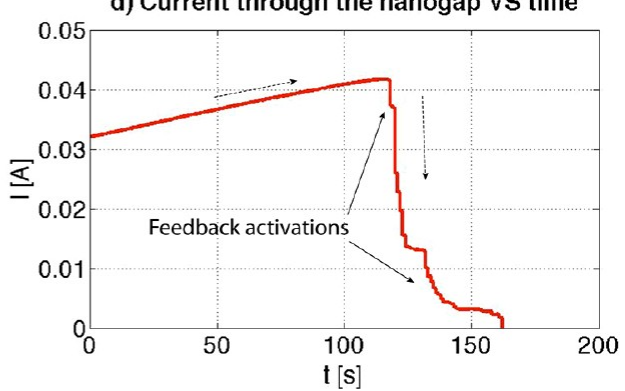

Figure 3. (a) Graph of the resistance versus time obtained during the fabrication of a nanogap. The process has three phases: In the first one, at the beginning, the wire is subjected to an increasing voltage that just warms it up. Once a certain temperature is reached the electromigration can start and the resistance increases exponentially. In this phase, the second one, the system control is activated repeatedly lowering each time the applied voltage until the phase 3 that corresponds to the nanogap realization. (b) Zoom of phase 2: it shows multiple feedback activations. (c) Detail of two feedback activation control that reduces the applied voltage (OFF) of a percentage value inserted by user interface before the starting of the process. This method allows avoiding the resistance runaway and the melt-down of the junction. As a consequence, the dimensions of the realized nanogaps can be below $10 \mathrm{~nm}$. (d) Same graph of panel a showing current versus time.

Agilent B1500A semiconductor device analyzer has been used, whereas for Bacteriorhodopsin proteins, a Cascade Microtech Summit 11000 with MicroChamber shield probe station and a Hewlett-Packard 4156A semiconductor parameter analyzer have been used. Moreover, in the experiments with $\mathrm{RC}$, an $80 \mathrm{~W}$ tungsten lamp has been exploited (to irradiate the RCs, a distance of $12 \mathrm{~cm}$ has been kept to avoid an increase in the temperature of the proteins), and the probe station was inserted into a dark box. The characterizations done with BR did not need a dark box because of the Faraday shield: the MicroChamber, in fact, can prevent the chuck to undergo excessive noise, reduce stray capacitances, and eliminate electromagnetic and electrostatic interferences, thus providing a dark, almost noise-free, measurement environment. An electronic circuit has been designed for the BR's photocharacterization to switch remotely on and off the green, red, and blue LEDs. The LEDs are put directly into the MicroChamber, and only small signal cables are routed to the circuit, which lies outside of the chamber itself, through a resilient slot that prevents the entrance of ambient light. Furthermore, the circuit implements an exponential decay of the LEDs current to avoid abrupt variations and induced noise due to mechanical switching. Addition- ally, two optical filters were exploited to cut out the Gaussian tails of the spectrum, keeping a very low noise-floor in the measurements: 480/30 (FITC-EX) for the blue LED and 700/75 (FITC-EM) for the red one. The LEDs were bought by Farnell-Switzerland.

\section{EXPERIMENTS AND RESULTS}

The realized structures consist in a silicon chip (see Figure 2) containing eight wires that will be electromigrated, becoming nanogaps. The aforementioned electromigration phenomenon has been controlled by a system with a feedback reaction, able to avoid the melting of the junction by modulating the applied voltage once the process of breaking is started, as shown in Figure 3. Once the gap is formed, the first step is checking if the process was successful, resulting in the formation of an under $10 \mathrm{~nm}$ gap, that is, a structure useful to analyze molecules. To reach this goal, an SEM imaging was used; Figure 4 shows one of the smallest nanogap realized by authors. 


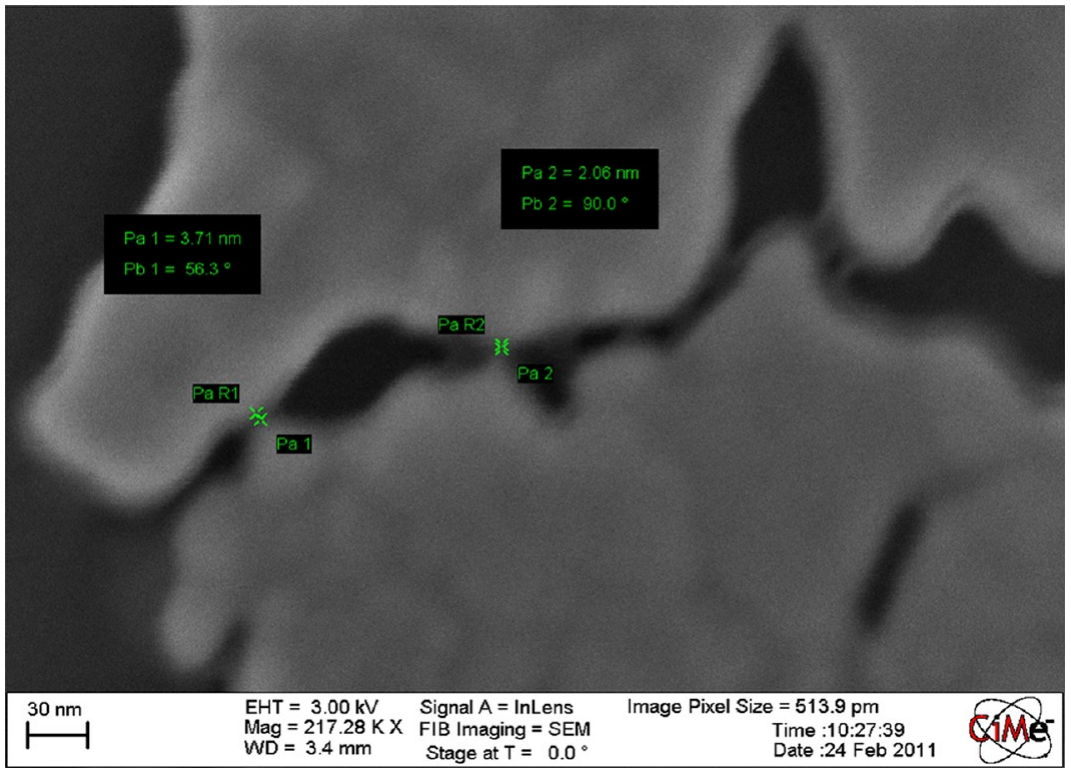

Figure 4. SEM measurements of an electromigrated gold nanogap. It is possible to see two of the smallest obtained nanogaps.

The selected probes are then exploited to realize metalmolecule-metal junctions by inserting proteins that have to be analyzed as in Figure 5. In this work, two different solutions

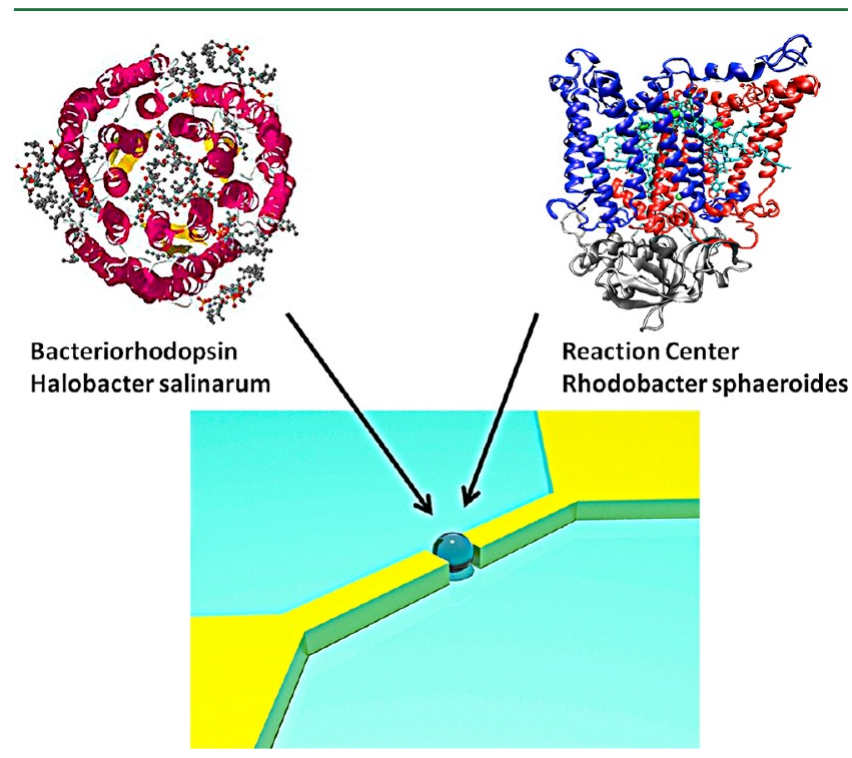

Figure 5. Schematic overview of the metal-molecule-metal junction: the glass sphere represents the molecule position when it is inserted in nanogap probes (in yellow).

have been prepared and inserted in nanogaps: one for RCs and one for Bacteriorhodopsin, respectively. The metal-moleculemetal systems have a different duration according to the proteins' survival capacity at room temperature and under ambient light. In case of RC, the activity is only 1 day, whereas for BRs, which are stronger, it can be a whole week. This is fundamental to perform repetitive measurements.

As was previously explained, to avoid any light-induced excitation, we placed a $1 \mu \mathrm{L}$ drop of $\mathrm{RC}$ solution in a nanogap probe under dark conditions. After waiting $10 \mathrm{~min}$, until the complete drying of the solution drop, the photoinduced electrical measurements can start. To show clearly the effect of light, we have performed $I-V$ measurements in two batches: the first, serving to characterize the current response of the protein to an applied voltage input in the absence of light stimuli, and the second, to characterize the current response of the protein to light radiation, in the visible field. In both cases, the applied voltage was in the -4 to $+4 \mathrm{~V}$ range (see Section 2.4 of the Materials and Methods, photoinduced voltage acquisition). Figure 6 describes the behavior of RC under dark

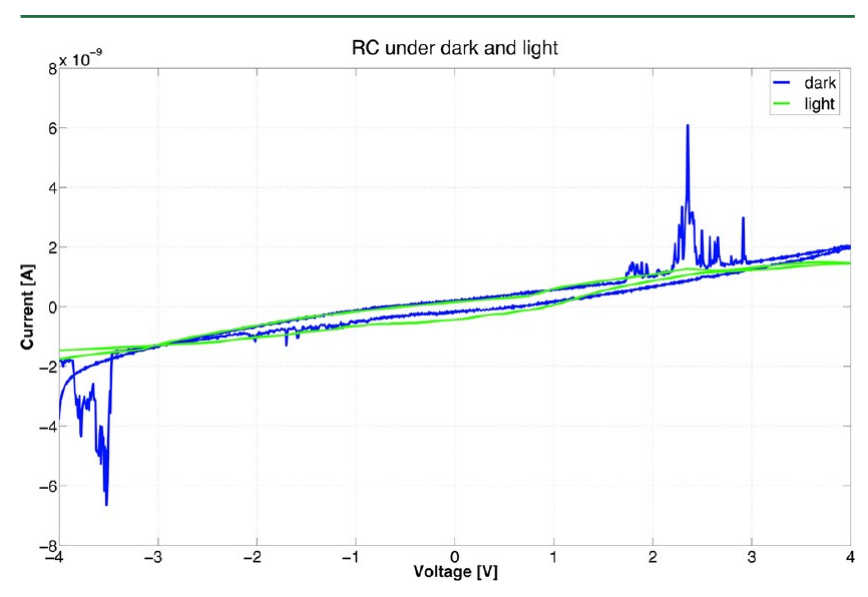

Figure 6. $I-V$ characterization of RC in between nanogap under light and dark. Several peaks are visible under dark because the applied voltage induces electron transfer inside the RC. In a first phase, under dark, there are no carriers in the excited level, but as soon as the applied voltage overcomes the excitation barrier, a resonant tunneling response can be recorded: in particular, at $2.5 \mathrm{~V}$ is shown a resonance peak. Under light irradiation, photons cause charge separation inside $\mathrm{RC}$ molecules and the current transmission is not allowed.

and light; the result is very close to the one obtained in ref 63 . However, in that case, the measurements were performed using STM on a RC Langmuir-Blodgett film, but the principle of electron tunneling and protein excitation was the same. Without applied light, several peaks are visible with a resonance voltage of $2.5 \mathrm{~V}$, which is not present if the protein is illuminated. The electron-transfer process inside the $\mathrm{RC}^{64}$ causes the resonance. It is then possible to excite the protein also under dark, by applying the correct voltage. Moreover, the 
voltage value at which the peak is recorded corresponds to the sum of energy required by the protein for electron transfer, that is, $800 \mathrm{mV}$, and the one to overcome the tunneling barrier between the protein itself and the nanogap electrodes (that is related to the measurement system setup). The curve recorded in light has no peaks because the light induces charge separation and the protein behaves as an open circuit, preventing electron transit.

AFM characterizations of the previously electrically analyzed probe have been performed in contact mode, and the result is shown in Figure 7. The AFM image shows a cluster of RC molecules located in the middle of the gap bridging it and creating the metal-molecule-metal junction.

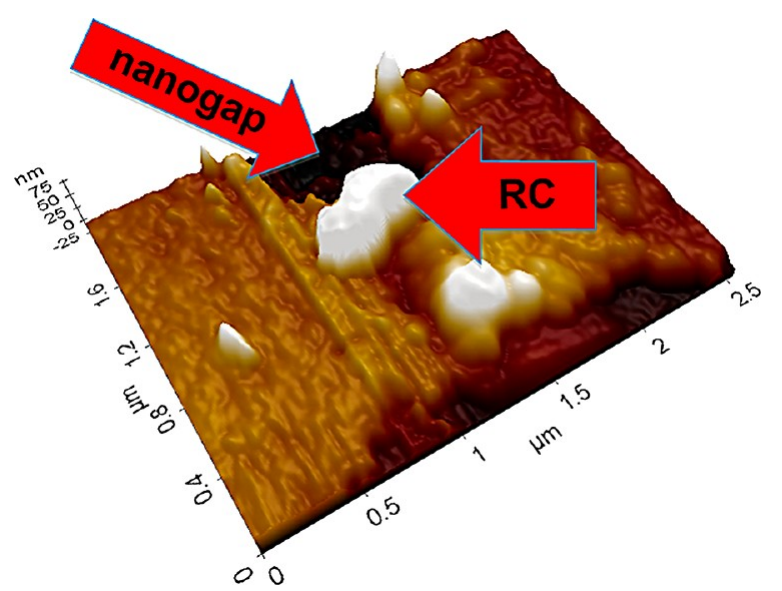

Figure 7. Representative AFM image of reaction centers in nanogap: the RC bridging the nanogap electrode is clearly visible.

Bacteriorhodopsin proteins have been solved in deionized water at different concentrations (see Section 2.1). In the dark, $1 \mu \mathrm{L}$ drop of solution was deposited on the nanogap sample; after $5 \mathrm{~min}$ of waiting time, $2 \mathrm{~V}$ voltage was applied between the gap, always maintaining the probe in the dark; this is a fundamental step for anchoring BR in the oriented manner to the nanoelectrodes. An electronic circuit has been designed to switch on and off remotely three LEDs of different colors: red, green, and blue.

Knowing that just the light around $570 \mathrm{~nm}$ (the green one, peak of BR absorbance) excites the proteins; for limiting light spectra in the correct range and cutting the Gaussian tails of the wavelengths, particular filters have been inserted ahead the red and the blue LEDs (see the Materials and Methods, photoinduced voltage acquisition). Switching on and off the LED light, photovoltage response of BR has been recorded through a signal analyzer, and the data have been elaborated with an "ad hoc" Labview program.

In Figure 8, the recorded photovoltage response of metalmolecule-metal junctions with the lower concentrated solution (i.e., $1 \mu \mathrm{g}$ of proteins in $100 \mu \mathrm{L}$ of DI water) is shown. Once $\mathrm{BR}$ proteins were exposed to the three light sources different wavelengths, it was possible to record an excitation response only from the green light, as was expected. The steep response of the protein to the light being switched on is clearly visible. The driving circuit exponentially reduces the current flowing into the LED, thus lowering emitted light until its complete turning off after $8 \mathrm{~s}$. The protein closely follows these variations until a threshold lighting level is reached. At this point, the

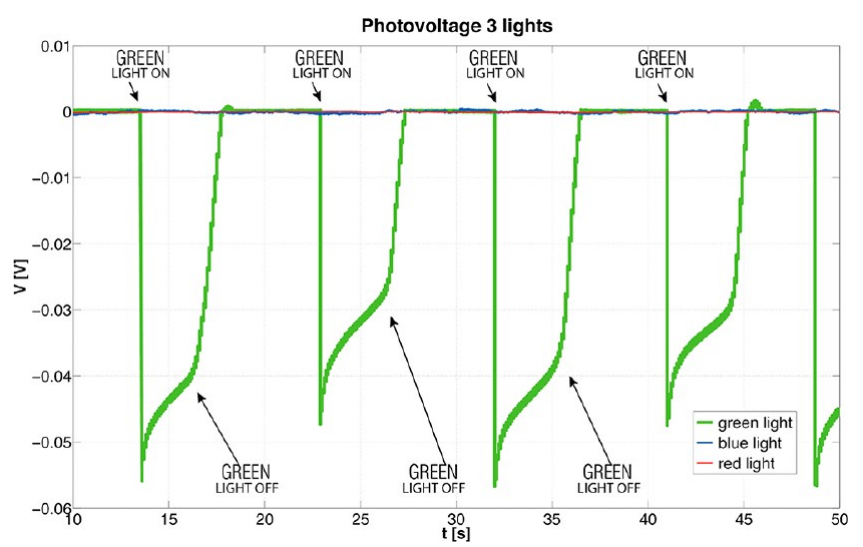

Figure 8. Different behavior of BR under three LEDs' light irradiation. Please notice that even if the red and blue lights are almost indistinguishable, the red light gives no response, whereas under blue light a noise has been recorded because of the broadband. Only the green one shows a sensitive reaction once the light is switched on and off. Note that the blue curve has been shifted of $0.2 \mathrm{~V}$ to bring it at zero. The presence of the offset in the blue curve can be explained also as some kind of photoeffect on metal electrodes.

protein very quickly starts behaving again as an open circuit showing a small overshoot.

It is interesting to consider how many proteins are required for the observed photovoltage. Considering that BR proteins are well-fitted to the nanogap size and well-oriented, we can estimate the potentials at left and right electrodes, resulting from the BR dipole induced by light. As a consequence, it is possible to calculate the value of photovoltage as

$$
\varphi=\frac{q a}{4 \pi \varepsilon \varepsilon_{0} r^{2}}
$$

where $q$ is the electron charge, $a$ is distance of the dipole (that is $5 \mathrm{~nm}), r$ is distance from the center of the dipole to the electrode (it is reasonable to assume that BRs fill all of the gap and therefore $r=a / 2$ ), and $\varepsilon$ is dielectric constant (refractive index for $\mathrm{BR}$ is about 1.54 as in ${ }^{65}$ ). Therefore, simple calculations give the possible photovoltage value of about 50-60 mV. The observed effect can be a result of the charge movement in even single BR proteins; however, this statement indicates only that no more than one protein thick layer is required to observe the voltage response, but elementary units work in parallel, giving effect in current; therefore, because here we are just measuring the voltage response it can be several proteins, corresponding to the cross section of the nanogap, but the exact number cannot be indicated.

\section{CONCLUSIONS AND FUTURE WORK}

Metal-molecule-metal junction is the starting point for the realization of a novel series of bio-optoelectronic transistors based on light-sensitive proteins, in which inorganic materials are well-integrated with biologically active elements. ${ }^{66-68}$ In this work, we fabricated circuits and devices in which proteins have been placed in a nanogap, and we demonstrated their ability to maintain light-induced charge-transfer properties. It was possible to elicit resonance in $\mathrm{RC}$ in response to a voltage application, whereas, under light, the charge separation induced in the photoactive protein was shown to avoid any current peak. Excitation of BR brought about similar results that are consistent with previous reports. ${ }^{69}$ AFM and SEM measurements allowed a more complete analysis of the experiments 
and, through verification of the obtained results, proved the presence of the molecules bridging nanogaps. In conclusion, we have demonstrated the RC capability as a switching element, if excited by proper voltage application. It has been shown that the optimal sensitivity of the metal-molecule-metal junction with $\mathrm{BR}$ is at $570 \mathrm{~nm}$ wavelength. However, reproducibility of the results of electrical testing is related to many factors as: number of adsorbed molecules, their position in the junction, and their possible motion. As a consequence, authors need to work more against these dynamic characteristics with statistical analysis based on photovoltage and photocurrent acquisitions. The exploitation of BRs opens a huge number of possible future works because they are candidates for the conversion of solar to electrical energy. We are still working on BR at different concentrations to validate different photocurrent amplitude response primarily considering the nanogap dimensions in which proteins are inserted.

\section{AUTHOR INFORMATION}

\section{Corresponding Author}

*E-mail: alice.dimonte@iit.it.

\section{Notes}

The authors declare no competing financial interest.

\section{ACKNOWLEDGMENTS}

We would like to thank Prof. Massimo Trotta from University of Bari for the preparation of RCs and for the support in treating proteins. For the SEM measurements, we acknowledge Dr. Fabienne Brodard of EPFL in Switzerland. The research has been funded by the project i-IronIC, financed with a grant from the Swiss Nano-Tera.ch initiative, and evaluated by the Swiss National Science Foundation.

\section{REFERENCES}

(1) Keane, Z. K.; Ciszek, J. W.; Tour., J. M.; Natelson, D. Nano Lett. 2006, 6, 1518-1521.

(2) Moth-Poulsen, K.; Bjørnholm, T. Nat. Nanotechnol. 2009, 4, $551-556$.

(3) Zhu, X.-Y. Surf. Sci. Rep. 2004, 56, 1-83.

(4) Hendriksen, B. L. M.; Martin, F.; Qi, Y.; Mauldin, C.; Vukmirovic, N.; Ren, J.; Wormeester, H.; Katan, A. J.; Altoe, V.; Aloni, S.; Frechet, J. M. J.; Wang, L. -W.; Salmeron, M. Nano Lett. 2011, 11, 4107-4112.

(5) Snyder, J. L.; Clark, A., Jr.; Fang, D. Z.; Gaborski, T. R.; Striemer, C. C.; Fauchet, P. M.; McGrath, J. L. J. Membr. Sci. 2011, 369, 119129.

(6) Carrara, S.; Riley, D. J.; Bavastrello, V.; Stura, E.; Nicolini, C. Sens. Actuators, B 2005, 105, 542-548.

(7) Haick, H.; Cahen, D. Prog. Surf. Sci. 2008, 83, 217-261.

(8) Tian, J. -H.; Liu, B.; Li, X.; Yang, Z. -L.; Ren, B.; Wu, S.-T.; Tao, N.; Tian, Z.-Q. J. Am. Chem. Soc. 2006, 128, 14748-14749.

(9) Facci, P.; Erokhin, V.; Carrara, S.; Nicolini, C. Proc. Natl. Acad. Sci. U.S.A. 1996, 93, 10556-10559.

(10) Carrara, S.; Bavastrello, V.; Ram, M. K.; Nicolini, C. Thin Solid Films 2006, 510, 229-264.

(11) Erokhin, V.; Carrara, S.; Amenitch, H.; Bernstorff, S.; Nicolini, C. Nanotechnology 1998, 9, 228-236.

(12) Carrara, S.; Ricci, D.; Di Zitti, E.; Di Fabrizio, E.; Altissimo, M.; Tormen, M. Mater. Lett. 2006, 60, 3682-3685.

(13) Fang, C.; Quan, q.; Liang, R.; Zhongyun, W.; Zhongfan, L. App. Phys. Lett. 2005, 86, 123105-123108.

(14) Li, C. Z.; He, H. X.; Tao, N. J. App. Phys. Lett. 2000, 77, 39953997.

(15) Kim, J.-H.; Moon, H.; Yoo, S.; Choi, Y.-K. Small 2011, 7, 22102216.
(16) Song, H.; Kim, Y.; Jeong, H.; Reed, M. A.; Lee, T. J. App. Phys. 2011, 109, 102419-102423.

(17) Morpurgo, A. F.; Marcus, C. M.; Robinson, D. B. Appl. Phys. Lett. 1999, 74, 2084-2086.

(18) Black, J. R. IEEE Trans. Electron Devices 1969, 4, 338-348.

(19) Motto, P.; Dimonte, A.; Rattalino, I.; Demarchi, D.; Piccinini, G.; Civera, P. Nano. Res. Lett. 2012, 7, 113-122.

(20) Xu, J.; Bhattacharya, P.; Varo, G. Biosens. Bioelectron. 2004, 19, $885-892$.

(21) Lee, T.-H.; Gonzales, J. I.; Zheng, J.; Dickson, R. M. Acc. Chem. Res. 2005, 38, 534-541.

(22) Kong, J.; Lu, Z.; Lvov, Y. M.; Desamero, R. Z. B.; Frank, H. A.; Rusling, J. F. J. Am. Chem. Soc. 1998, 120, 7371-7372.

(23) Lukashev, E. P.; Nadtochenko, V. A.; Permenova, E. P.; Sarkisov, O. M.; Rubin, A. B. Dokl. Biochem. Biophys. 2007, 415, 696702.

(24) Stowell, M. H. B.; McPhilips, T. M.; Rees, D. C.; Soltis, S. M.; Abresch, E.; Feher, G. Science 1997, 276, 812-816.

(25) Labahn, A.; Paddock, M. L.; McPherson, P. H.; Okamura, M. Y.; Feher, G. J. Phys. Chem. 1994, 98, 3417-3423.

(26) Lanyi, J. K. Biochemistry (Moscow) 2001, 66, 1192-1196.

(27) Kondo, M.; Iida, K.; Dewa, T.; Tanaka, H.; Ogawa, T.; Nagashima, S.; Nagashima, K. V. P.; Shimada, K.; Hashimoto, H.; Gardiner, A. T.; Cogdell, R. J.; Nango, M. Biomacromolecules 2012, 13, $432-438$.

(28) Xu, J.; Lu, Y.; Liu, B.; Xu, C.; Kong, J. Solid State Electrochem. 2007, 11, 1689-1695.

(29) Kong, J.; Sun, W.; Wu, X.; Deng, J.; Lu, Z.; Lvov, Y.; Desamero, R. Z. B.; Frank, H. A.; Rusling, J. F. Bioelectrochem. Bioeng. 1999, 48, 101-107.

(30) Aribe, A.; Khaled, M. J. Microelectromech. Syst. 2011, 20, 800810 .

(31) Nagy, K. Biochem. Biophys. Res. Commun. 1978, 85, 383-390.

(32) Varò, G. Acta Biol. Acad. Sci. Hung. 1981, 32, 301-310.

(33) Nicolini, C.; Erokhin, V.; Paddeu, S.; Sartore, M. Nanotechnology 1998, 9, 223-227.

(34) Tiede, D. M.; Mueller, P.; Dutton, P. L. Biochim. Biophys. Acta 1982, 81, 191-210.

(35) Gopher, A.; Blatt, Y.; Schönfeld, M.; Okamura, M. Y.; Feher, G.; Montal, M. Biophys. J. 1985, 48, 311-320.

(36) Popovic, Z. D.; Kovacs, G. J.; Vincett, -P.S.; Dutton, P. L. Chem. Phys. Lett. 1985, 116, 405-410.

(37) Popovic, Z. D.; Kovacs, G. J.; Vincett, -P.S.; Alegria, G.; Dutton, P. L. Chem. Phys. 1986, 110, 227-237.

(38) Popovic, Z. D.; Kovacs, G. J.; Vincett, -P.S.; Alegria, G.; Dutton, P. L. Biochim. Biophys. Acta, Bioenerg. 1986, 851, 38-48.

(39) Erokhin, V.; Feigin, L.; Kayushina, R.; Lvov, Y.; Kononenko, A.; Knox, P.; Zakharova, N. Stud. Biophys. 1987, 122, 231-236.

(40) Fujihira, M.; Sakomura, M.; Kamei, T. Thin Solid Films 1989, $180,43-50$.

(41) Alegria, G.; Dutton, P. L. Biochim. Biophys. Acta 1991, 1057, 239-257.

(42) Erokhin, V.; Facci, P.; Nicolini, C. Biosens. Bioelectron. 1995, 10, $25-34$.

(43) Oesterhelt, D.; Stoeckenius, W. Nat. New Biol. 1971, 233, 149152.

(44) Hayward, S. B.; Grano, D. A.; Glaeser, R. M.; Fisher, K. A. Proc. Natl. Acad. Sci. U.S.A. 1978, 75, 4320-4324.

(45) Bamberg, E.; Dencher, N. A.; Fahr, A.; Heyn, M. P. Proc. Natl. Acad. Sci. U.S.A. 1981, 78, 7502-7506.

(46) Lukashev, E. P.; Zaitsev, S. Y.; Kononenko, A. A.; Zubov, V. P. Stud. Biophys. 1989, 132, 111-118.

(47) Birge, R. R. Annu. Rev. Phys. Chem. 1990, 41, 683-733.

(48) Mikayama, T.; Iida, K.; Suemori, Y.; Dewa, T.; Miyashita, T.; Nango, M.; Gardiner, A. T.; Cogdell, R. J. J. Nanosci. Nanotechnol. 2009, 9, 97-107.

(49) Nakamura, C.; Hasegawa, M.; Yasuda, Y.; Miyake, J. Appl. Biochem. Biotechnol. 2000, 84, 401-408. 
(50) Nakamura, C.; Hasegawa, M.; Nakamura, N.; Miyake, J. Biosens. Bioelectron. 2003, 18, 599-603.

(51) Zhao, J.; Yonglong, Z.; Baohong, L.; Chunhe, X.; Jilie, K. Biosens. Bioelectron. 2002, 17, 711-718.

(52) Trammell, S. A.; Wang, L.; Zullo, J. M.; Shashidhar, R.; Lebedev, N. Biosens. Bioelectron. 2004, 19, 1649-1655.

(53) Lebedev, N.; Trammell, S. A.; Spano, A.; Lukashev, E.; Griva, I.; Schnur, J. J. Am. Chem. Soc. 2006, 128, 12044-12045.

(54) Mikayama, T.; Iida, K.; Suemori, Y.; Dewa, T.; Miyashita, T.; Nango, M.; Gardiner, A. T.; Cogdell, R. J. J. Nanosci, Nanotechnol. 2009, 9, 97-107.

(55) Ahmadi, M.; Yeow, J. T. W. Biosens. Bioelectron. 2011, 26, 21712176.

(56) Bhattacharya, P.; Xu, J.; Varò, G.; Marcy, D. L.; Birg, R. R. Opt. Lett. 2002, 27, 839-841.

(57) Shin, J.; Bhattacharya, P.; Yuan, H. C.; Ma, Z.; Varò, G. Opt. Lett. 2007, 32, 500-502.

(58) Jin, Y.; Honig, T.; Ron, I.; Friedman, N.; Sheves, M.; Cahen, D. Chem. Soc. Rev. 2008, 37, 2422-2432.

(59) Griep, M. H.; Walczak, K. A.; Winder, E. M.; Lueking, D. R.; Friedrich, C. R. Biosens. Bioelectron. 2010, 25, 1493-1497.

(60) Isaacson, R. A.; Lendzian, F.; Abresch, E. C.; Lubitz, W.; Feher, G. Biophys. J. 1995, 69, 311-322.

(61) Zahid, F.; Paulsson, M.; Datta, S. Electrical Conduction through Molecules. In Advanced Semiconductors and Organic Nano-Techniques; Morkoc, H., Ed.; Academic Press: San Diego, CA, 2003.

(62) Hansen, K.; Laegsgaard, E.; Stensgaard, I.; Besenbacher, F. Phys. Rev. B 1997, 56, 2208-2220.

(63) Johnson, E. T.; Nagarajan, V.; Zazubovich, V.; Riley, K.; Small, G. J.; Parson, W. W. Biochem. 2003, 42, 13673-13683.

(64) Facci, P.; Erokhin, V.; Nicolini, C. Thin Solid Films 1994, 243, 403-406.

(65) Zhang, C.; Song, Q. W.; Ku, C.; Gross, R. B.; Birge, R. R. Opt. Lett. 1994, 19, 1409-1411.

(66) Chu, L. K.; Yen, C. W.; El-Sayed, M. A. Biosens. Bioelectron. 2010, 26, 620-626.

(67) Das, R.; Kiley, P. J.; Segal, M.; Norville, J.; Yu, A. A.; Wang, L.; Trammell, S. A.; Reddick, L. E.; Kumar, R.; Stellacci, F.; Lebedev, N.; Schnur, J.; Bruce, B. D.; Zhang, S.; Baldo, M. Nano Lett. 2004, 4, 1079-1083.

(68) Den Hollander, M. J.; Magis, J. G.; Fuchsenberger, P.; Aartsma, T. J.; Jones, M. R.; Frese, N. R. Langmuir 2011, 27, 10282-10294.

(69) Wasielewski, M. R. Chem. Rev. 1992, 92, 435-461. 